\title{
A Young Planetary Mass Companion to the Nearby M Dwarf VHS J125601.92-125723.9
}

\author{
B. Gauza ${ }^{1}$, V. J. S. Béjar ${ }^{1}$, A. Pérez-Garrido ${ }^{2}$, M. R. Zapatero \\ Osorio $^{3}$, N. Lodieu ${ }^{1}$, R. Rebolo ${ }^{1}$, E. Pallé ${ }^{1}$ and G. Nowak ${ }^{1}$ \\ ${ }^{1}$ Instituto de Astrofísica de Canarias (IAC), Calle Vía Láctea s/n, E-38200 La Laguna, \\ Tenerife, Spain \\ email: bgauza@iac.es vbejar@iac.es \\ ${ }^{2}$ Dpto. Física Aplicada, Universidad Politécnica de Cartagena, Campus Muralla del Mar, \\ Cartagena, Murcia E-30202, Spain \\ ${ }^{3}$ Centro de Astrobiología (CSIC-INTA), Ctra. Ajalvir km 4, 28850, Torrejón de Ardoz, \\ Madrid, Spain
}

\begin{abstract}
We have recently identified a young, very red $\left(J-K_{s}=2.47 \mathrm{mag}\right)$ late L-type companion at $8.06 " \pm 0.03 "$ ( $\sim 102 \mathrm{AU})$ from a previously unrecognized M dwarf. We determined the parallactic distance of the system to be $12.7 \pm 1.0 \mathrm{pc}$. Non-detection of lithium and the kinematics of the primary allowed us to constrain the age of the system in the range of $150-300$ Myr. By comparison with theoretical evolutionary models we derived a mass of $73_{-15}^{+20} M_{\mathrm{Jup}}$ for the primary, at around the substellar mass regime and $11.2_{-1.8}^{+9.7} M_{\mathrm{Jup}}$ for the secondary, near the deuterium burning mass limit.
\end{abstract}

Keywords. stars: brown dwarfs - stars: imaging - infrared: planetary systems - stars: individual (VHS J125601.92-125723.9)

\section{Introduction}

Young L dwarfs have recently been identified, either free-floating (Peña Ramírez et al. 2012; Zapatero Osorio et al. 2014) or as companions to stars (e.g., Wahhaj et al. 2011; Bowler et al. 2013; Chauvin et al. 2015). They were found to share similar photometric and spectral properties that are distinct from those of typical late-type objects of the field population. Some of these peculiarities have been attributed to low surface gravities and cloudy atmospheres, as expected at early evolutionary stages, less than several hundred million years (Cruz et al. 2009; Allers \& Liu 2013). They have very red colors $(J-$ $K_{s}>2 \mathrm{mag}$ ), $J$-band absolute magnitudes fainter than their old, field counterparts, and show distinctive spectral features such as, for example, a sharply peaked, triangularshaped continuum in the $H$-band and weaker $\mathrm{Na}$ I and $\mathrm{K}$ I lines.

Recent studies have revealed a strong resemblance between the young $\mathrm{L}$ dwarfs and directly imaged planetary mass companions (e.g., 2MASS 1207-39 b, Chauvin et al. 2005, HR 8799 bcde, Marois et al. 2010, GJ 504 b Kuzuhara et al. 2013). They have similar near-infrared (near-IR) colors and absolute magnitudes, overlapping effective temperature regimes of $\sim 1000-1500 \mathrm{~K}$ and masses of a few to a few tens of Jupiter masses Spectroscopic and photometric studies of young substellar objects can provide information on the physical properties of gas giant exoplanets found by transit and radial velocity surveys, in particular, on the characteristics and composition of their complex atmospheres. Here we describe the physical properties of the recently identified nearby, young binary system VHS 1256-1257: spectral types of the two components, distance, age, luminosities, masses, and effective temperatures. 


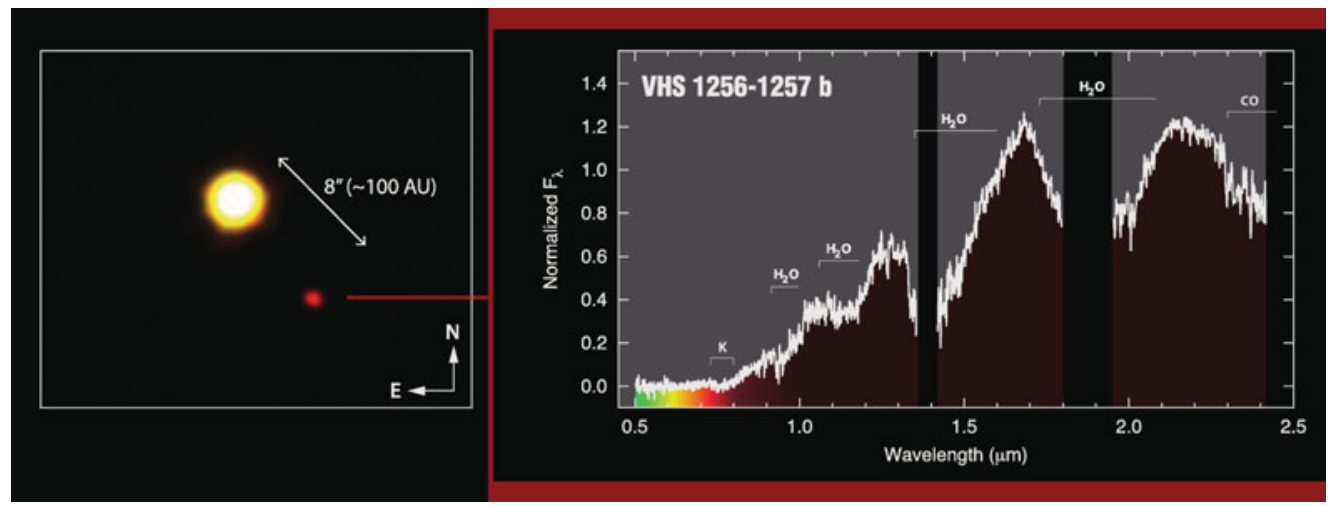

Figure 1. Left: image of the planetary mass companion at 8.06" $\pm 0.03 "(\sim 102$ AU) from the low-mass star VHS 1256. This false color image was obtained from the $Y, J$ and $K_{s}$ bands images taken with the VISTA telescope. Right: Low-resolution optical and near-infrared spectra of the companion.

Table 1. Selected measurements and parameters of VHS 1256-1257 components.

\begin{tabular}{|c|c|c|c|c|c|c|c|c|}
\hline & SpType & $\begin{array}{l}\mu_{\alpha} \cos \delta \\
{[\mathrm{mas} / \mathrm{yr}]}\end{array}$ & $\begin{array}{c}\mu_{\delta} \\
{[\mathrm{mas} / \mathrm{yr}]}\end{array}$ & $\begin{array}{c}J \\
{[\mathrm{mag}]}\end{array}$ & $\begin{array}{c}J-K_{s} \\
{[\mathrm{mag}]}\end{array}$ & $\underset{\left(L_{\mathrm{bol}} / L_{\odot}\right)}{\log }$ & $\begin{array}{c}\text { Mass } \\
{\left[M_{\mathrm{Jup}}\right]}\end{array}$ & $\begin{array}{l}T_{\text {eff }} \\
{[\mathrm{K}]}\end{array}$ \\
\hline Primary & $\mathrm{M} 7.5 \pm 0.5$ & $-281.5 \pm 5.3$ & $-205.5 \pm 15.2$ & $11.02 \pm 0.02$ & $0.97 \pm 0.04$ & $-3.14 \pm 0.10$ & $73_{-15}^{+20}$ & $2620 \pm 140$ \\
\hline Secondary & $\mathrm{L} 7.0 \pm 1.5$ & $-275.4 \pm 5.3$ & $-198.4 \pm 15.2$ & $17.14 \pm 0.02$ & $2.47 \pm 0.03$ & $-5.05 \pm 0.22$ & $11.2_{-1.8}^{+9.7}$ & $880_{-110}^{+140}$ \\
\hline
\end{tabular}

\section{Identification of the system}

Using the VISTA Hemisphere Survey (VHS, McMahon et al. 2013) data and the 2MASS (Skrutskie et al. 2006), we carried out a search for high proper motion objects, by cross-matching both catalogs. The search focused on objects that had moved at least 2 arcsec and a maximum of 30 arcsec from 2MASS to VHS. The time baseline between the two surveys is typically about $12 \mathrm{yr}$, which gives proper motions of approximately 0.15-3.0 $\operatorname{arcsec} \mathrm{yr}^{-1}$. Over the common area between 2MASS and VHS we found more than 6000 objects with $J=11-17$ mag and proper motion higher than 150 mas yr $^{-1}$. We have searched for common proper motion pairs and multiples among these objects with proper motions consistent within 40 mas yr $^{-1}$ in both $\mu_{\alpha}$ and $\mu_{\delta}$. VHS 1256-1257 system was one of the identified candidates.

A VISTA image of the pair is shown in Fig. 1. The companion is located at a projected angular separation of $8.06 \pm 0.03$ arcsec. This separation corresponds to a projected orbital separation of $102 \pm 9$ AU at the estimated distance of the system. The two components share a common proper motion, which significantly differs from the proper motion of background stars. The $\mu_{\alpha} \cos \delta$ and $\mu_{\delta}$ measured from the VHS and 2MASS positions of the sources and other selected parameters of the two components are listed in Table 1.

\section{Physical properties of the components}

To determine the spectral types, we used low-resolution optical and near-IR spectra obtained using GTC/OSIRIS and NTT/SofI instruments, respectively. We classified the objects through visual comparison of the spectra with a set of field dwarf spectral templates, in the optical and near-IR separately, with known young L dwarfs in the near-IR, and using the spectral indices established by Allers \& Liu (2013). We adopted an M7.5 \pm 0.5 and L7.0 \pm 1.5 spectral types for the primary and secondary, respectively. 

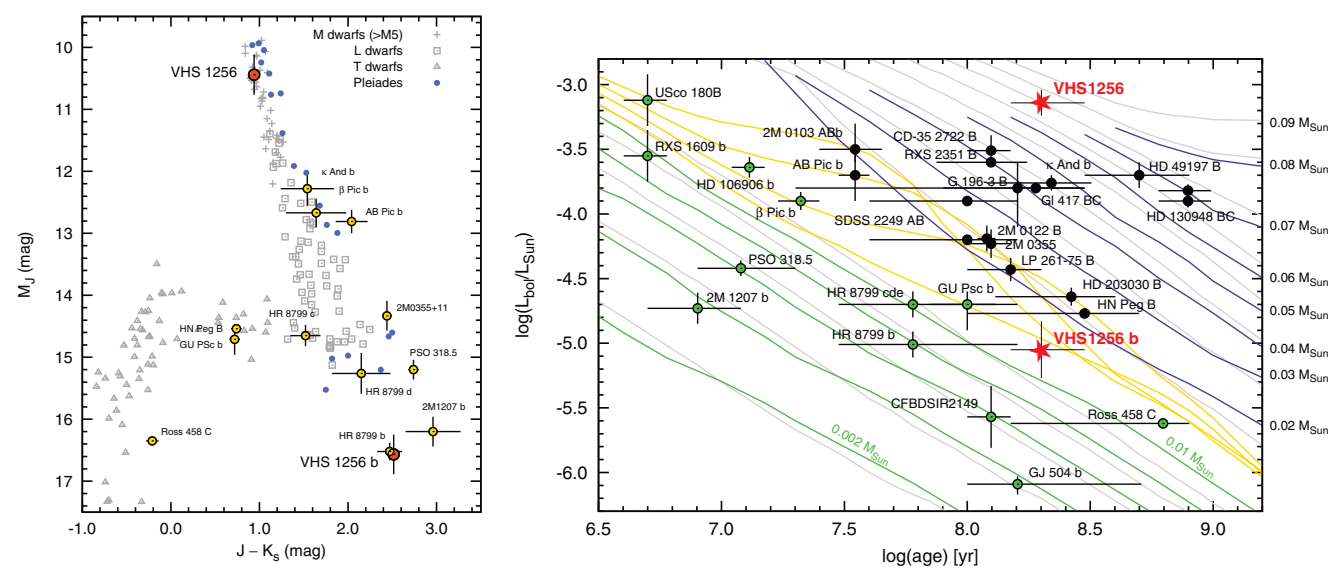

Figure 2. Left: $M_{J}$ vs. $J-K_{s}$ color-magnitude diagrams comparing the two components of VHS 1256-1257 with field M, L, and T dwarfs with parallax measurements, known young substellar objects and the least-massive Pleiades members. Right: Luminosity and age of the two components compared with evolutionary tracks from the cloudy atmosphere models of Saumon \& Marley (2008), the BT-Settl models (Allard \& Barman 2004) and known young substellar objects. The Saumon \& Marley models are plotted in color, gray curves correspond to BT-Settl.

The near-IR spectrum of the secondary (right panel of Fig. 1) shows a peaked triangular shape of the $H$-band continuum, and other spectral features recognized as hallmarks of low surface gravity and youth, like weak Na I and K I lines. The optical spectrum of the primary also shows weaker alkali lines than of the field counterparts, but no Li I at 670.82 $\mathrm{nm}$ at a limit of $\mathrm{pEW}<30 \mathrm{~m} \AA$.

Using the VISTA data and a set of follow-up images collected from March to December, 2014 using NTT/SofI, IAC80/CAMELOT and WHT/LIRIS instruments, we have obtained a first measurement of the trigonometric parallax. The absolute parallax is $\pi=78.79 \pm 6.4$ mas, which translates into a distance of the system of $12.7 \pm 1.0 \mathrm{pc}$. To measure the heliocentric radial velocity of VHS 1256-1257 we employed high spectral resolution $(R \sim 40000)$ UVES data and the cross-correlation method against the M6V star GJ 406, which has a known, constant radial velocity of $v_{r}=19.5 \pm 0.1 \mathrm{~km} \mathrm{~s}^{-1}$ (Nidever et al. 2002). GJ 406 was also observed with the VLT/UVES instrument with similar spectral resolution, data is available in the ESO Archive. The measured heliocentric radial velocity is $v_{r}=-1.4 \pm 5.0 \mathrm{~km} \mathrm{~s}^{-1}$, Having the measurements of proper motion, parallax, and radial velocity we could determine the three components of the Galactic space velocity, $U, V$, and $W$, obtaining $-9.4 \pm 2.0,-16.4 \pm 3.0$ and $-8.5 \pm 3.9 \mathrm{~km} \mathrm{~s}^{-1}$, for each component, respectively.

The non-detection of Li I imposes a lower limit on the mass of the primary of $0.055-$ $0.060 M_{\odot}$ and also imposes a lower limit on the age of the system, since objects of similar spectral type in the Pleiades cluster (age $\sim 120 \mathrm{Myr}$ ) have fully preserved this element. Theoretical evolutionary models predict that objects with $T_{\text {eff }} \sim 2600 \mathrm{~K}$ (which is the corresponding effective temperature of an M7.5 dwarf) have preserved their Li content at ages $<150 \mathrm{Myr}$. The galactic $U V W$ velocities indicate that the system probably belongs to the Local Association, whose members have estimated ages of 10-300 Myr. In conclusion, based on the absence of Li I in the primary and the likely membership in the Local Association, we adopt a range of 150-300 Myr for the age of the VHS 1256-1257 system.

In Fig. 2 we compare $M_{J}$ versus $J-K_{s}$ and luminosities at the adopted range of ages of the two components of the pair with field M, L and T dwarf sequence (on the 
color-mag diagram), with the Saumon \& Marley (2008) and BT-Settl models evolutionary tracks (on the right panel) and with known young substellar objects. On the $M_{J}, J-$ $K_{s}$ CMD, the secondary, VHS 1256 b is located at almost the same position as the HR $8799 \mathrm{~b}$ planet. Using bolometric corrections we derive luminosities and compared with theoretical models to derive masses, temperatures and $\log g$. For the primary, we obtained a mass of $73_{-15}^{+20} M_{\mathrm{Jup}}$, close to the boundary between stars and brown dwarfs. The effective temperature and $\log g$ found from the models are $2620 \pm 140 \mathrm{~K}$ and $5.05 \pm$ 0.10 dex, respectively. For the companion, we obtained a mass of $11.2_{-1.8}^{+9.7} M_{\mathrm{Jup}}$, indicating that the object is near the mass limit at which the onset of deuterium fusion occurs. Given the uncertainty in the mass determination, it is currently unclear whether the object is above or below it. The effective temperature and $\log g$ of the companion obtained from the evolutionary models are $880_{-110}^{+140} \mathrm{~K}$ and $4.24_{-0.10}^{+0.35}$ dex.

\section{Final remarks}

VHS $1256-1257 \mathrm{~b}$ is among the nearest currently known planetary mass companions detected by direct imaging. Moreover, it is one of the very few young, extremely red L dwarfs with age constrained within a narrow range, given by the likely belonging to the Local Association and the absence of $\mathrm{Li}$ I in the primary. The $T_{\text {eff }}$ of $\sim 900 \mathrm{~K}$ determined from evolutionary models based on the luminosity does not seem to be consistent with the expected $T_{\text {eff }}$ range of field dwarfs of similar spectral type and with the absence of methane, which is expected to appear in atmosphere cooler than $1400 \mathrm{~K}$. Following Barman et al. (2011) the formation of clouds with substantial vertical thickness and non-equilibrium chemistry in a low-gravity object like VHS 1256-1257 b could provide an explanation of the apparent high atmospheric temperature $(>1500 \mathrm{~K})$ as compared to cooling track effective temperature predictions $(900-1000 \mathrm{~K})$. Since it is a relatively nearby and bright object near the deuterium-burning limit, it becomes one of the most promising targets to study the application of the deuterium test. From the masses and separation of the components we estimate the orbital period to be about $3900 \mathrm{yr}$. Assuming a circular orbit with a face-on orientation, the displacement caused by the orbital motion would be from 4 to 13 mas/yr, which will become feasible to measure in the next few years using precise astrometric observations.

\section{References}

Allard, F. \& Barman, T. S. 2004, IAU Symposium, Vol. 213, 119

Allers, K. N. \& Liu, M. C. 2013, ApJ, 772, 79

Barman, T. S., Macintosh, B., Konopacky, Q. M., \& Marois, C. 2011, ApJL, 735, L39

Bowler, B. P., Liu, M. C., Shkolnik, E. L., \& Dupuy, T. J. 2013, ApJ, 774, 55

Chauvin, G., Lagrange, A. M., Dumas, C., et al., 2005, A $\& A$ A, 438, L25

Chauvin, G., Vigan, A., Bonnefoy, M., et al., 2015, A\& $A, 573,127$

Cruz, K. L., Kirkpatrick, J. D., \& Burgasser, A. J. 2009, AJ, 137, 3345

Deacon, N. R. \& Hambly, N. C 2007, A\&A, 468, 163

Eggen, O. J. 1992, AJ, 104, 2141

Eisenbeiss, T., Ammler-von Eiff, M., Roell, T., et al., 2013, A\&A, 556, 53

Gauza, B., Béjar, V. J., Pérez-Garrido, A., et al., 2015, ApJ, 804, 96

Kuzuhara, M., Tamura, M., Kudo, T., et al., 2013, ApJ, 774, 11

Marois, C., Zuckerman, B., Konopacky, Q. M., et al., 2010, Nature, 468, 1080

McMahon, R. G., Banerji, M., Gonzalez, E., et al., 2013, The Messenger, 154, 35

Peña Ramírez, K., Béjar, V. J. S.., Zapatero Osorio, M. R., et al., 2012, ApJ, 754, 30

Saumon, D. \& Marley, M. S. 2008, ApJ, 689, 1327S 
Skrutskie, M. F., Cutri, R. M., Stiening, R., et al., 2006, AJ, 131, 1163

Nidever, D. L., Marcy, G. W., Butler, R. P., Fischer, D. A., \& Vogt, S. S. 2002, ApJS, 141, 503

Wahhaj, Z., Liu, M. C., Biller, B. A., et al., 2011, ApJ, 729, 139

Zapatero Osorio, M. R., Gálvez Ortiz, M. C., Bihain, G., et al., 2014, A\&6A, 568, A77

\section{Discussion}

Author: A question by Eric Mamajek was regarding the age upper limit. He asked if the restriction of $300 \mathrm{Myr}$ which we adopt comes only from the probable membership of the system to the Local Association.

Author: Having measured the proper motion, parallax and radial velocity of the primary, we could determine its $U V W$ galactic velocities. The kinematics are consistent with a range where several young moving groups overlap, but some of them like the $\beta$ Pictoris, Taurus-Auriga or TW Hya can be rejected since they are younger than $100 \mathrm{Myr}$, and this age is incompatible with the non-detection of lithium in the atmosphere of the primary. However, the kinematic and spectroscopic properties of VHS 1256-1257 appear consistent with membership in Local Association Group, a coherent kinematic stream of young stars, all below 300 Myr (Eggen 1992). The Local Association is considered by some authors as having much wider spread of ages, up to 1 Gyr, we thus agree that this upper age constraint is not as strong as the lower limit of $150 \mathrm{Myr}$ coming from the nondetection of Li I. Nevertheless, we see various spectral features of both of the components suggesting an age considerably younger than 1 Gyr. The weak alkali lines, peculiar red $J-K_{s}$ color and peaked, triangular shape of the H-band continuum are recognized as hallmarks of very young ages, below $500 \mathrm{Myr}$. We would like to highlight, that in case of VHS 1256 its not the case since we know the system is older than $150 \mathrm{Myr}$.

Additionally, from tests using the LACEwING moving group identification code, Adric Riedel has found (private communication) that the system has a significant probability of being a member of the Hercules-Lyra moving group (250 Myr, Eisenbeiss et al. 2013), imposing an age constraint consistent with our estimates.

Author: As mentioned in the original paper (Gauza et al. 2015) the primary and the secondary were cataloged in 2MASS with designations 2MASS J125602.15-125721.7 and 2MASS J125601.83-125727.6, respectively. The primary was also listed as SIPS 1256-1257

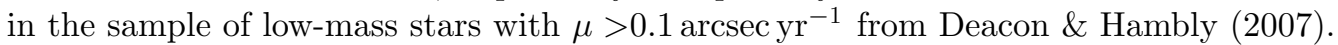
Eric has also noticed, what we didn't realize before, that the primary was also detected by Luyten as a HPM star and listed in his catalog as LP736-15. 